\title{
Self-energy of image states on copper surfaces
}

\author{
I. Sarria \\ Materia Kondentsatuaren Fisika Saila, Zientzi Fakultatea, Euskal Herriko Unibertsitatea, \\ 644 Posta Kutxatila, 48080 Bilbo, Spain \\ J. Osma \\ Materialen Fisika Saila, Kimika Fakultatea, Euskal Herriko Unibertsitatea, 1072 Posta Kutxatila, \\ 20080 Donostia, Spain \\ E. V. Chulkov \\ Materialen Fisika Saila, Kimika Fakultatea, Euskal Herriko Unibertsitatea, 1072 Posta Kutxatila, \\ 20080 Donostia, Spain \\ and Donostia International Physics Center (DIPC) and Centro Mixto CSIC-UPV/EHU, Donostia, Spain \\ J. M. Pitarke \\ Materia Kondentsatuaren Fisika Saila, Zientzi Fakultatea, Euskal Herriko Unibertsitatea, 644 Posta Kutxatila, \\ 48080 Bilbo, Spain \\ and Donostia International Physics Center (DIPC) and Centro Mixto CSIC-UPV/EHU, Donostia, Spain \\ P. M. Echenique \\ Materialen Fisika Saila, Kimika Fakultatea, Euskal Herriko Unibertsitatea, 1072 Posta Kutxatila, \\ 20080 Donostia, Spain \\ and Donostia International Physics Center (DIPC) and Centro Mixto CSIC-UPV/EHU, Donostia, Spain
}

(Received 9 June 1999)

\begin{abstract}
We report extensive calculations of the imaginary part of the electron self-energy in the vicinity of the (100) and (111) surfaces of $\mathrm{Cu}$. The quasiparticle self-energy is computed by going beyond a free-electron description of the metal surface, either within the $G W$ approximation of many-body theory or with inclusion, within the $G W \Gamma$ approximation, of short-range exchange-correlation effects. Calculations of the decay rate of the first three image states on $\mathrm{Cu}(100)$ and the first image state on $\mathrm{Cu}(111)$ are also reported, and the impact of both band structure and many-body effects on the electron relaxation process is discussed.
\end{abstract}

[S0163-1829(99)03940-5]

\section{INTRODUCTION}

It is well known that an electron outside a metal surface experiences an effective potential with the asymptotic image form $V_{i m}=-1 / 4\left(z-z_{0}\right), z_{0}$ representing the image-plane position. If the bulk band structure projected into the surface presents a band gap near the vacuum level $\left(E_{\mathrm{v}}=0\right)$, an electron with energy $E<0$ can be trapped in the potential well created on the vacuum side of the surface by the gap and the image potential. The long-range character of the image potential gives rise to a series of unoccupied Rydberg-like states, which converge towards the vacuum level. ${ }^{1,2}$ These so-called image states were first detected experimentally ${ }^{3,4}$ by the techniques of inverse photoemission, ${ }^{5,6}$ and the first high-resolution measurements of image states were made by the use of two-photon photoemission (2PPE) ${ }^{7-11}$

Image-potential states are localized in the vacuum region of the surface. Hence, they are almost decoupled from bulk states and are expected to have much longer lifetimes than bulk excitations. Lifetimes of image states had been determined from their spectral width in 2PPE spectroscopy, and the advent of time-resolved 2PPE (TR-TPPE) has enabled a direct measurement of image-state lifetimes on the (100) and
(111) surfaces of silver ${ }^{12-14}$ and copper. ${ }^{15-19}$

Calculations of image-state lifetimes were reported in Refs. 20 and 21, within a many-body free-electron description of the metal surface and with the use of simplified models to approximate both initial and final electronic states and, also, the screened Coulomb interaction. Later on, the role that crystal-induced surface states, not present within a freeelectron description of the solid, play in the decay of image states was investigated. ${ }^{22,23}$ Self-consistent many-body calculations of image-state lifetimes that go beyond a freeelectron description of the metal surface have been carried out only very recently on copper ${ }^{24}$ and lithium ${ }^{25}$ surfaces.

In this paper, we report extensive calculations of the imaginary part of the image-electron self-energy in the vicinity of the (100) and (111) surfaces of copper, which we compute within the $G W$ approximation of many-body theory. ${ }^{26}$ Single-particle wave functions are obtained by solving the Schrödinger equation with a realistic one-dimensional model potential, and the screened interaction is evaluated within the random-phase approximation (RPA). ${ }^{27}$ Then, we present the results of calculations of the lifetime of the first three image states on $\mathrm{Cu}(100)$ and the first image state on $\mathrm{Cu}(111)$. These calculations are carried out within a $G W$-RPA description of 
the self-energy and, also, with use of the so-called $G W \Gamma$ approximation, ${ }^{28,29}$ which includes short-range exchangecorrelation (XC) effects not present in the $G W$-RPA. We also consider simplified models for the description of both the electronic wave functions and the screened interaction, we discuss the impact of band structure and many-body effects on image-state lifetimes, and account for the potential variation parallel to the surface through the introduction of the effective mass. We present systematic investigations of the role that all quantities involved in the evaluation of the electron self-energy play in the coupling of image states with the solid. We find that a detailed description of these quantities is of crucial importance in the understanding of the origin and magnitude of decay rates of image states. Finally, differences between decay rates of image states on the (100) and (111) surfaces of $\mathrm{Cu}$ are investigated, and we focus our attention on the role that the various available bulk and surface states play in the electron relaxation process.

Unless otherwise is stated, atomic units are used throughout, i.e., $e^{2}=\hbar=m_{e}=1$.

\section{THEORY}

Take an inhomogeneous electron system. The damping rate of an excited electron in the state $\psi_{0}(\mathbf{r})$ with energy $E_{0}$ is obtained as the projection of the imaginary part of the electron self-energy, $\Sigma\left(\mathbf{r}, \mathbf{r}^{\prime}, E_{0}\right)$, over the state itself:

$$
\tau^{-1}=-2 \int d \mathbf{r} \int d \mathbf{r}^{\prime} \psi_{0}^{*}(\mathbf{r}) \operatorname{Im} \Sigma\left(\mathbf{r}, \mathbf{r}^{\prime} ; E_{0}\right) \psi_{0}\left(\mathbf{r}^{\prime}\right)
$$

We consider a periodic-film model of the solid. The film is taken to be translationally invariant in the plane of the surface, which is assumed to be normal to the $z$ axis, and departure of motion along the surface from free-electron behavior is accounted for through the introduction of the effective mass. Hence, single-particle wave functions are taken to be of the form

$$
\psi_{0}(\mathbf{r})=\frac{1}{\sqrt{A}} \phi_{0}(z) e^{\mathrm{i}} \mathbf{k}_{\|} \cdot \mathbf{r}_{\|}
$$

with energies

$$
E_{0}=\varepsilon_{0}+\frac{\mathbf{k}_{\|}^{2}}{2 m_{0}} .
$$

The wave function $\phi_{0}(z)$ and energy $\varepsilon_{0}$ describe motion normal to the surface, $\mathbf{k}_{\|}$is a wave vector parallel to the surface, $A$ is the normalization area, and $m_{0}$ is the effective mass. Introduction of Eq. (2) into Eq. (1) yields the following expression for the damping rate:

$$
\tau^{-1}=-2 \int d z \int d z^{\prime} \phi_{0}^{*}(z) \operatorname{Im} \Sigma\left(z, z^{\prime} ; \mathbf{k}_{\|}, E_{0}\right) \phi_{0}\left(z^{\prime}\right),
$$

where $\Sigma\left(z, z^{\prime} ; \mathbf{k}_{\|}, E_{0}\right)$ represents the two-dimensional Fourier transform of the electron self-energy.

In the so-called $G W$ approximation, one considers only the first-order term in a series expansion of the self-energy in terms of the screened interaction,

$$
\begin{aligned}
\Sigma\left(z, z^{\prime} ; \mathbf{k}_{\|}, E_{0}\right)= & \int_{-\infty}^{\infty} \frac{d E}{2 \pi} \int \frac{d \mathbf{q}_{\|}}{(2 \pi)^{2}} W\left(z, z^{\prime} ; \mathbf{q}_{\|}, E\right) \\
& \times \mathrm{i} G\left(z, z^{\prime} ; \mathbf{k}_{\|}-\mathbf{q}_{\|}, E_{0}-E\right),
\end{aligned}
$$

and after replacing the Green function $G\left(z, z^{\prime} ; \mathbf{k}_{\|}, \omega\right)$ by that of noninteracting electrons, one finds

$$
\begin{aligned}
\operatorname{Im} \Sigma\left(z, z^{\prime} ; \mathbf{k}_{\|}, E_{0}\right)= & \sum_{f} \phi_{f}^{*}\left(z^{\prime}\right) \phi_{f}(z) \int \frac{d \mathbf{q}_{\|}}{(2 \pi)^{2}} \\
& \times \operatorname{Im} W\left(z, z^{\prime} ; \mathbf{q}_{\|}, E_{0}-E_{f}\right),
\end{aligned}
$$

where

$$
E_{f}=\varepsilon_{f}+\frac{\left(\mathbf{k}_{\|}+\mathbf{q}_{\|}\right)^{2}}{2 m_{f}}
$$

The sum in Eq. (6) is extended over a complete set of final states $\phi_{f}(z)$ with energy $\varepsilon_{f}\left(E_{F} \leqslant E_{f} \leqslant E_{0}\right), E_{F}$ is the Fermi energy, and $W\left(z, z^{\prime} ; \mathbf{q}_{\|}, \omega\right)$ is the two-dimensional Fourier transform of the screened interaction:

$$
\begin{aligned}
W\left(z, z^{\prime} ; \mathbf{q}_{\|}, \omega\right)= & \mathbf{v}\left(z, z^{\prime} ; \mathbf{q}_{\|}\right)+\int d z_{1} \int d z_{2} \mathbf{v}\left(z, z_{1} ; \mathbf{q}_{\|}\right) \\
& \times \chi\left(z_{1}, z_{2} ; \mathbf{q}_{\|}, \omega\right) \mathbf{v}\left(z_{2}, z^{\prime} ; \mathbf{q}_{\|}\right),
\end{aligned}
$$

with $\mathrm{v}\left(z, z^{\prime} ; \mathbf{q}_{\|}\right)$and $\chi\left(z, z^{\prime} ; \mathbf{q}_{\|}, \omega\right)$ representing the bare Coulomb interaction,

$$
\mathrm{V}\left(z, z^{\prime} ; \mathbf{q}_{\|}\right)=\frac{2 \pi}{q_{\|}} e^{-q_{\|}\left|z-z^{\prime}\right|},
$$

and the density-response function, respectively.

Within RPA, $\chi\left(z, z^{\prime} ; \mathbf{q}_{\|}, \omega\right)$ satisfies the integral equation

$$
\begin{aligned}
\chi\left(z, z^{\prime} ; \mathbf{q}_{\|}, \omega\right)= & \chi_{0}\left(z, z^{\prime}, \mathbf{q}_{\|}, \omega\right) \\
& +\int d z_{1} \int d z_{2} \chi_{0}\left(z, z_{1}, \mathbf{q}_{\|}, \omega\right) \\
& \times \vee\left(z_{1}, z_{2} ; \mathbf{q}_{\|}\right) \chi\left(z_{2}, z^{\prime}, \mathbf{q}_{\|}, \omega\right),
\end{aligned}
$$

where $\chi_{0}\left(z, z^{\prime}, \mathbf{q}_{\|}, \omega\right)$ represents the density-response function of noninteracting electrons, ${ }^{30}$

$$
\begin{aligned}
\chi_{0}\left(z, z^{\prime}, \mathbf{q}_{\|}, \omega\right)= & \sum_{l, l^{\prime}} \phi_{l}(z) \phi_{l^{\prime}}^{*}(z) \phi_{l^{\prime}}\left(z^{\prime}\right) \phi_{l}^{*}\left(z^{\prime}\right) \\
& \times \int \frac{d \mathbf{k}_{\|}}{(2 \pi)^{2}} \frac{\theta\left(E_{F}-E_{l}\right)-\theta\left(E_{F}-E_{l^{\prime}}\right)}{E_{l}-E_{l^{\prime}}+(\omega+\mathrm{i} \eta)},
\end{aligned}
$$

with

$$
E_{l}=\varepsilon_{l}+\frac{\mathbf{k}_{\|}^{2}}{2}
$$

and

$$
E_{l^{\prime}}=\varepsilon_{l^{\prime}}+\frac{\left(\mathbf{k}_{\|}+\mathbf{q}_{\|}\right)^{2}}{2}
$$

and $\theta(x)$ being the Heaviside step function. 
In the so-called $G W \Gamma$ approximation, which includes $\mathrm{XC}$ effects not present in the $G W$-RPA, the electron self-energy is of the $G W$ form, i.e., it is given by Eq. (5), but with an effective screened interaction,

$$
\begin{aligned}
W\left(z, z^{\prime} ; \mathbf{q}_{\|}, \omega\right)= & \mathbf{v}\left(z, z^{\prime} ; \mathbf{q}_{\|}\right)+\int d z_{1} \int d z_{2} \\
& \times\left[\mathbf{v}\left(z_{1}, z_{2} ; \mathbf{q}_{\|}\right)+K_{x c}\left(z_{1}, z_{2} ; \mathbf{q}_{\|}, \omega\right)\right] \\
& \times \chi\left(z_{1}, z_{2} ; \mathbf{q}_{\|}, \omega\right) \mathbf{v}\left(z_{2}, z^{\prime} ; \mathbf{q}_{\|}\right),
\end{aligned}
$$

where

$$
\begin{aligned}
\chi\left(z, z^{\prime} ; \mathbf{q}_{\|}, \omega\right)= & \chi_{0}\left(z, z^{\prime}, \mathbf{q}_{\|}, \omega\right) \\
& +\int d z_{1} \int d z_{2} \chi_{0}\left(z, z_{1}, \mathbf{q}_{\|}, \omega\right) \\
& \times\left[\mathbf{v}\left(z, z_{1} ; \mathbf{q}_{\|}\right)+K_{x c}\left(z_{1}, z_{2} ; \mathbf{q}_{\|}, \omega\right)\right] \\
& \times \chi\left(z_{2}, z^{\prime}, \mathbf{q}_{\|}, \omega\right) .
\end{aligned}
$$

Here, $K_{x c}\left(z, z^{\prime} ; \mathbf{q}_{\|}, \omega\right)$ represents the two-dimensional Fourier transform of the XC kernel $K_{x c}\left(\mathbf{r}, \mathbf{r}^{\prime} ; \omega\right)$, which accounts through Eqs. (14) and (15) for the reduction in the electronelectron interaction due to the existence of short-range XC effects associated to the probe electron and to screening electrons, respectively. In the static limit $(\omega \rightarrow 0)$, densityfunctional theory (DFT) (Ref. 31) shows that ${ }^{32}$

$$
K_{x c}\left(\mathbf{r}, \mathbf{r}^{\prime} ; \omega \rightarrow 0\right)=\left[\frac{\delta^{2} E_{x c}[n]}{\delta n(\mathbf{r}) \delta n\left(\mathbf{r}^{\prime}\right)}\right]_{n_{0}(\mathbf{r})},
$$

where $E_{x c}[n]$ represents the XC energy functional and $n_{0}(\mathbf{r})$ is the actual density of the electron system. In the localdensity approximation (LDA), the XC kernel is approximated by a contact $\delta$ function, and one finds

$$
K_{x c}\left(z, z^{\prime} ; \mathbf{q}_{\|}, \omega \rightarrow 0\right)=\left[\frac{d^{2} E_{x c}(n)}{d n^{2}}\right]_{n_{0}(z)} \delta\left(z-z^{\prime}\right),
$$

where $E_{x c}[n]$ now represents the XC energy of a homogeneous electron gas of density $n{ }^{33}$ Introduction of this static XC kernel into Eq. (15) represents an adiabatic extension of the LDA to finite frequencies, and yields the so-called timedependent LDA (TDLDA). ${ }^{34}$

The single-particle wave functions $\phi_{i}(z)$ entering Eqs. (4), (6), and (11) are simply eigenfunctions of a one-electron Hamiltonian. In the particular case of the RPA, $\phi_{i}(z)$ are self-consistent eigenfunctions of the one-electron Hartree Hamiltonian, and within TDLDA they are obtained by solving the Kohn-Sham equation of DFT with use of the LDA $\mathrm{XC}$ potential,

$$
V_{x c}(z)=\left[\frac{d E_{x c}(n)}{d n}\right]_{n_{0}(z)} .
$$

Neither the Hartree self-consistent eigenfunctions nor the LDA wave functions produce the correct imagelike asymptotic potential behavior on the vacuum side of the surface.

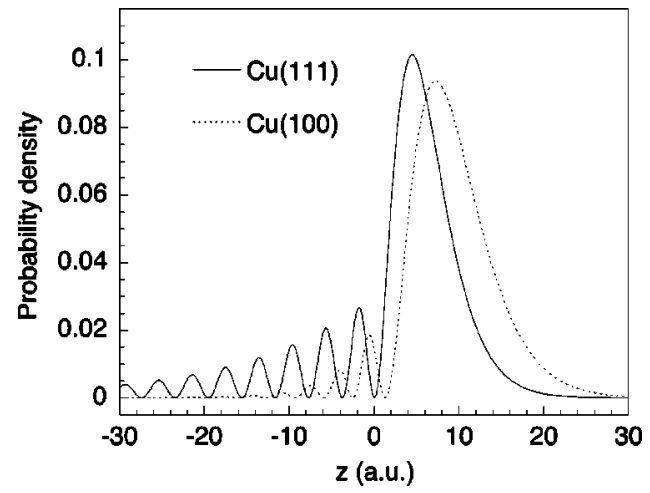

FIG. 1. Probability density of the $n=1$ image state on $\mathrm{Cu}(100)$ (dotted line) and $\mathrm{Cu}(111)$ (solid line), as obtained with use of the one-dimensional model potential of Ref. 35. The crystal edge $(z$ $=0$ ) has been chosen to be located half an interlayer spacing beyond the last atomic layer. Full circles represent the atomic positions. In the case of $\mathrm{Cu}(111)$, the matching at the surface occurs at minimum amplitude.

For a realistic description of the metal surface, we solve for $\phi_{i}(z)$ a single-particle time-independent Schrödinger equation with the one-dimensional model potential suggested in Ref. 35, which approaches, far outside the surface, the classical image potential. This one-dimensional potential has four adjustable parameters, which are chosen so as to reproduce the width and position of the energy gap at the $\bar{\Gamma}$ point $\left(\mathbf{k}_{\|}=0\right)$ and, also, the binding energies of both the $n=0$ crystal-induced surface state at $\bar{\Gamma}$ and the first $(n=1)$ imagepotential induced state. Probability amplitudes of the $n=1$ image state on $\mathrm{Li}(110)$ and $\mathrm{Cu}(100)$, as obtained with use of this model potential, have been found to be in excellent agreement with first principles pseudopotential ${ }^{35}$ and all-electron ${ }^{36}$ calculations, respectively.

\section{RESULTS AND DISCUSSION}

Input of our calculation of image-state decay rates, as given by Eq. (4), are the image-state wave function $\phi_{0}(z)$ and the image-electron self-energy $\Sigma\left(z, z^{\prime} ; \mathbf{k}_{\|}, E_{0}\right)$. We use films of up to 50 layers of atoms and 80 interlayer-spacing vacuum intervals, thereby ensuring that finite-slab effects are negligible. Image-state wave functions are taken to be eigenfunctions of the one-dimensional model Hamiltonian described above (MP). For the evaluation of the image-electron self-energy, we use in Eq. (6) either the MP wave functions or the self-consistent jellium LDA eigenfunctions of the oneelectron Kohn-Sham Hamiltonian without (J) and with (JG) the restriction that only final states with energy $\varepsilon_{f}$ lying below the projected band gap are allowed. The screened interaction entering Eq. (6) is evaluated either within the specular-reflection model (SRM) of Ritchie and Marusak, ${ }^{37}$ with use of the approximate surface response function of Persson and Zaremba (PZ) ${ }^{38}$ or within the self-consistent approaches of Eqs. (8) and (14) with the single-particle eigenstates entering Eq. (11) being MP wave functions.

Probability densities, $\left|\phi_{0}(z)\right|^{2}$, for the $n=1$ image state on the (100) and (111) surfaces of $\mathrm{Cu}$, as obtained with use of the one-dimensional model potential of Ref. 35, are represented in Fig. 1. In the case of $\mathrm{Cu}(100)$ (dotted line), the 

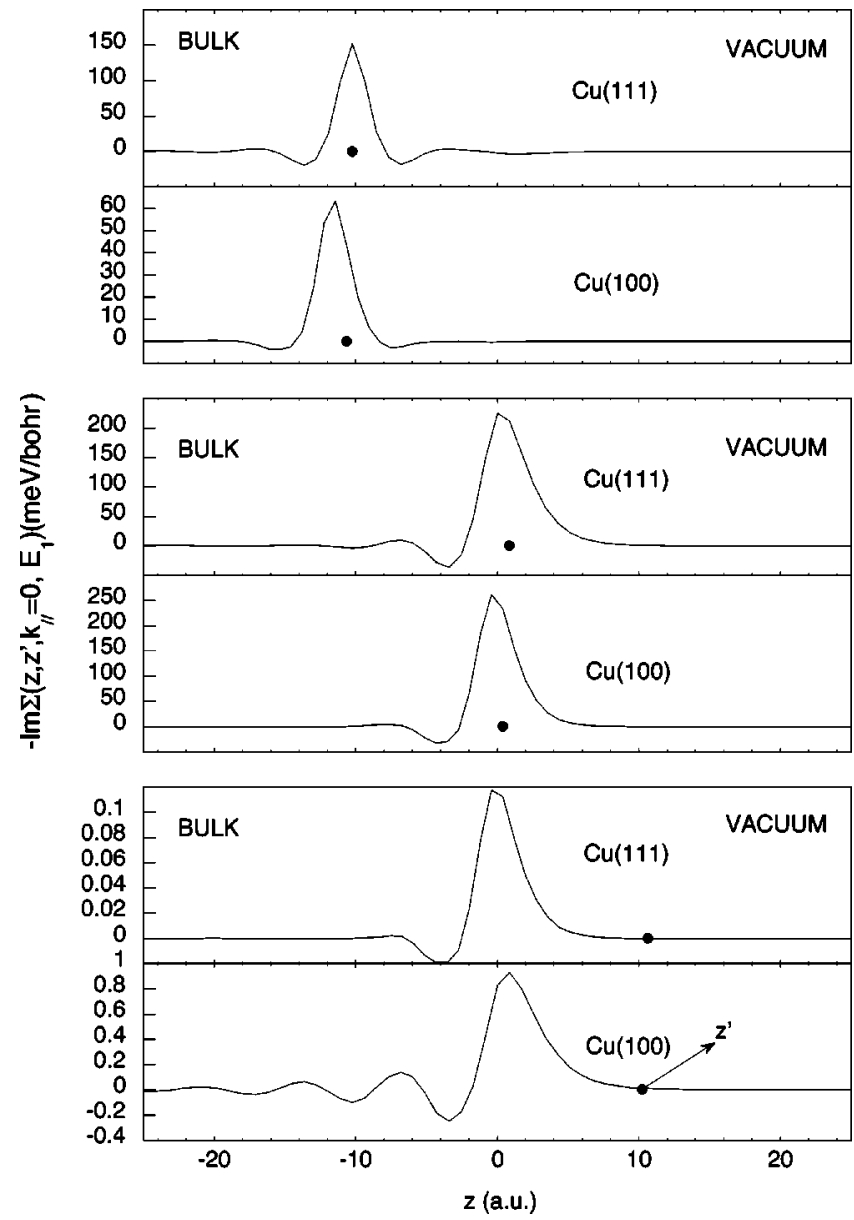

FIG. 2. Imaginary part of the $n=1$ image-electron $G W$-RPA self-energy $\operatorname{Im}\left[-\Sigma\left(z, z^{\prime} ; \mathbf{k}_{\|}=0, E_{0}\right)\right]$, versus $z$, in the vicinity of the (100) and (111) surfaces of $\mathrm{Cu}$, as obtained from Eq. (6) with use of MP wave functions both in Eq. (6) and Eq. (11) and with all effective masses set equal to the free-electron mass. $z^{\prime}$ is fixed at -5 (top panel), 0 (middle panel), and $5 \AA$ (bottom panel).

$n=1$ probability density has a maximum at $3.8 \AA$ outside the crystal edge $(z=0)$, which we choose to be located half a lattice spacing beyond the last atomic layer, and the penetration into the bulk $(z<0)$ crystal is found to be of $5 \%$. On the (100) surface of $\mathrm{Cu}$, the $n=1$ image state is close to the center of the projected band gap, ${ }^{10}$ which results in a very small penetration into the bulk. However, the $n=1$ image state on $\mathrm{Cu}(111)$ (solid line) is located right at the top of the gap, ${ }^{10}$ the solution in the bulk is an $s$-like wave function with the matching at the surface occurring at minimum amplitude, the maximum of the probability-density in the vacuum is closer from the surface, at $2.3 \AA$, than in the case of $\mathrm{Cu}(100)$, and the penetration into the bulk is found to be of $22 \%$. The first image state on $\mathrm{Cu}(100)$ and $\mathrm{Cu}(111)$ has binding energies of 0.57 and $0.82 \mathrm{eV}$, respectively.

Figure 2 shows full $G W$-RPA calculations of the imaginary part of the $n=1$ image-electron self-energy $\operatorname{Im}\left[-\Sigma\left(z, z^{\prime} ; \mathbf{k}_{\|}=0, E_{0}\right)\right]$ in the vicinity of the $(100)$ and (111) surfaces of $\mathrm{Cu}$, as obtained from Eq. (6) with use of MP wave functions both in Eq. (6) and Eq. (11) and with all effective masses set equal to the free-electron mass. The imaginary part of the electron self-energy is represented in this figure as a function of $z$ and for a fixed value of $z^{\prime}$. In the

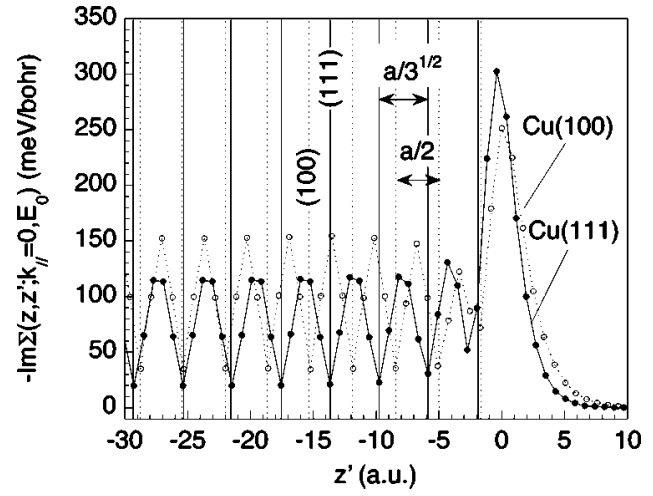

FIG. 3. Maximum of the imaginary part of the $n=1$ imageelectron $G W$-RPA self-energy $\operatorname{Im}\left[-\Sigma\left(z, z^{\prime} ; \mathbf{k}_{\|}=0, E_{0}\right)\right]$, versus $z^{\prime}$, in the vicinity of the (100) (dotted lines) and (111) (solid lines) surfaces of $\mathrm{Cu}$, obtained as in Fig. 2. Vertical dotted and solid lines represent the atomic positions along the (100) and (111) directions, respectively.

top panel, $z^{\prime}$ is fixed at about three atomic layers $\left(z^{\prime} \sim\right.$ $-5 \AA$ ) within the bulk, showing that $\operatorname{Im}(-\Sigma)$ has a maximum at $z=z^{\prime}$, as in the case of a homogeneous electron gas. When $z^{\prime}$ is fixed at the crystal edge $\left(z^{\prime} \sim 0\right)$, as shown in the middle panel of Fig. 2, we find that $\operatorname{Im}(-\Sigma)$ is still maximum at $z=z^{\prime}$, but the magnitude of this maximum is now enhanced. The bottom panel of Fig. 2 corresponds to $z^{\prime}$ being fixed at about three atomic layers $\left(z^{\prime} \sim 5 \AA\right)$ from the surface into the vacuum. In this case, the maximum magnitude of $\operatorname{Im}(-\Sigma)$ occurs at $z \sim 0$ rather than for $z=z^{\prime}$, showing a highly nonlocal behavior of the imaginary part of the electron self-energy at the surface. This nonlocality of the cusp of $\operatorname{Im}(-\Sigma)$ was also shown by Deisz et al. ${ }^{39}$ within a free-electron (jellium) description of the surface.

The magnitude of the maximum of $\operatorname{Im}(-\Sigma)$ is plotted in Fig. 3, as a function of $z^{\prime}$, showing that it is an oscillating function of $z^{\prime}$ within the bulk and reaches its highest value near the surface. The oscillatory behavior within the bulk is dictated by the periodicity of final-state wave functions $\phi_{f}(z)$ in the crystal, and the highest value near the crystal edge is the result of electron-hole pair creation taking place mainly in the vicinity of the surface. We note that the magnitude of $\operatorname{Im}(-\Sigma)$ near the surface is larger for $\mathrm{Cu}(111)$ than for $\mathrm{Cu}(100)$, although the band gap on $\mathrm{Cu}(111)$ extends below the Fermi level, thus the available phase space on this surface becomes highly restricted. However, while the crystal-induced $n=0$ surface state on $\mathrm{Cu}(100)$ is located outside the projected band gap and represents, therefore, a socalled surface resonance, the $n=0$ surface state on $\mathrm{Cu}(111)$ is located within the band gap and provides an important channel for the decay of image states. As a result, the imaginary part of the image-electron self-energy near the (111) surface of $\mathrm{Cu}$ is largely enhanced. This is illustrated in Fig. 4 , where contributions to the magnitude of the maximum $\operatorname{Im}(-\Sigma)$ of the $n=1$ image state on $\mathrm{Cu}(111)$ have been plotted separately, according to whether transitions to bulk states (dotted line) or to the $n=0$ surface state (dashed line) occur.

Now we focus on the evaluation of image-state lifetimes, and we set the wave vector of the image electron parallel to the surface, $\mathbf{k}_{\|}$, equal to zero. Coupling of the image state 


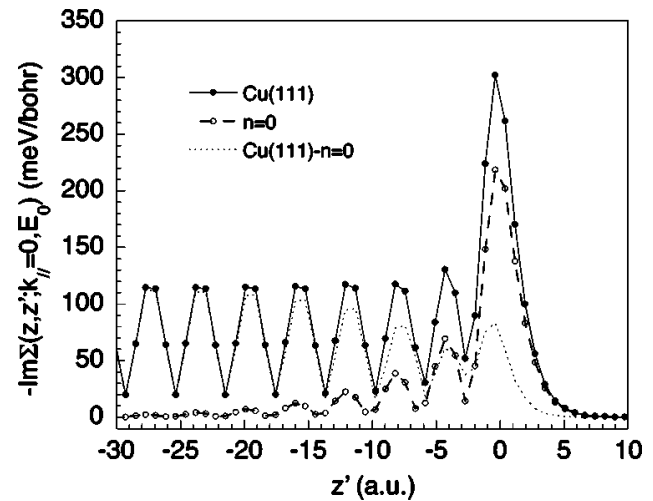

FIG. 4. As in Fig. 4, for the separate contributions to the magnitude of the maxiumum of $\operatorname{Im}[-\Sigma]$ of the $n=1$ image electron on $\mathrm{Cu}(111)$, according to whether transitions to bulk states (dotted line) or to the $n=0$ surface state (dashed line) occur.

with the crystal occurs through the penetration of the imagestate wave function into the solid and, also, through the evanescent tails of bulk states outside the crystal. Accordingly, we have calculated separately the various contributions to the damping rate by confining the integral in Eq. (4) to either bulk $(z<0)$ or vacuum $(z>0)$ coordinates:

$$
\tau^{-1}=\tau_{\text {bulk }}^{-1}+\tau_{\text {vac }}^{-1}+\tau_{\text {inter }}^{-1},
$$

where $\tau_{\text {bulk }}^{-1}, \tau_{\mathrm{vac}}^{-1}$, and $\tau_{\text {inter }}^{-1}$ represent bulk, vacuum, and interference contributions, respectively. The results of our calculations for the decay rate of the $n=1$ image state on the (100) and (111) surfaces of $\mathrm{Cu}$ are presented in Tables I, II, and III.

In order to investigate the impact of band structure effects on the damping rate of image states, we present in Table I the results of our full $G W$-RPA calculations for the decay rate of the $n=1$ image state on $\mathrm{Cu}(100)$, as obtained from Eq. (4) with use of either J, JG, or MP wave functions in Eq. (6), with use of MP wave functions in Eq. (11), and with all effective masses set equal to the free-electron mass. For $q_{\|}$ $>\sqrt{2\left(E_{0}-E_{g}\right)}\left(E_{g}\right.$ represents the bottom of the projected band gap) all final states with energy $E_{f}<E_{0}$ lie below the gap, thereby bulk and interference contributions to the decay rate remain nearly unaffected by this restriction. However, as the coupling of the image state with the crystal occurring through the tails of bulk states outside the crystal is expected to be dominated by vertical transitions $\left(\mathbf{q}_{\|} \sim 0\right)$, the vacuum contribution to the decay rate becomes noticeably smaller as

TABLE I. $G W$-RPA decay rates, in linewidth units (meV), of the $n=1$ image state on $\mathrm{Cu}(100)$, as obtained from Eqs. (4), (6), (8), and (10). Effective masses have been set equal to the freeelectron mass $\left(m_{f}=1\right)$. All wave functions entering Eqs. (4) and (11) have been chosen to be MP wave functions, and the final-state wave functions entering Eq. (6) have been taken to be either J, JG, or MP wave functions (see the text).

\begin{tabular}{clccl}
\hline \hline$\phi_{f}(z)$ & Bulk & Vacuum & Inter. & Total \\
\hline $\mathrm{J}$ & 21 & 58 & -12 & 67 \\
$\mathrm{JG}$ & 19.5 & 13 & -11 & 21.5 \\
$\mathrm{MP}$ & 24 & 14 & -16 & 22 \\
\hline \hline
\end{tabular}

TABLE II. Decay rates, in linewidth units (meV), of the $n=1$ image state on $\mathrm{Cu}(100)$, together with the experimentally determined decay rate of Refs. 18 and 19. All the wave functions entering Eqs. (4), (6), and (11) have been chosen to be MP wave functions. Effective masses have been set equal to either the freeelectron mass $\left(m_{f}=1\right)$ or to realistic values for all available final states $\left(m_{f} \neq 1\right)$. Five different models for the description of the screened interaction have been used: the specular reflection model $(\mathrm{SRM}),{ }^{37}$ the model of Persson and Zaremba (PZ), ${ }^{38}$ and three selfconsistent many-body approaches, $G W$-RPA, $G W$-TDLDA, and $G W \Gamma$-TDLDA.

\begin{tabular}{lcccccc}
\hline \hline$m_{f}$ & $W$ & Bulk & Vacuum & Inter. & Total & Exp. \\
\hline$=1$ & SRM & 18 & 3 & -4 & 17 & \\
$=1$ & PZ & & 55 & & 55 & \\
$=1$ & $G W$-RPA & 24 & 14 & -16 & 22 & \\
$=1$ & $G W$-TDLDA & 26.5 & 14 & -16 & 24.5 & \\
$=1$ & $G W \Gamma$-TDLDA & 23.5 & 14 & -16 & 21.5 & \\
$\neq 1$ & $G W$-RPA & 7 & 11.5 & -1 & 17.5 & \\
$\neq 1$ & $G W \Gamma$-TDLDA & 6.5 & 11.5 & -1 & 17 & 16.5 \\
\hline \hline
\end{tabular}

final states lying within the projected band gap are not allowed, and this restriction results in a total decay rate that is smaller by a factor of $\sim 3$. However, the decay rate is nearly insensitive to the actual choice of the one-particle wave functions entering Eq. (6), as long as only final states lying below the projected band gap are included in the jellium calculation. We have also performed calculations with use of either $\mathrm{J}$ or JG wave functions in Eq. (11), and have found that the decay rate is insensitive to the actual choice of the oneparticle wave functions entering Eq. (11). In the case of the (111) surface of $\mathrm{Cu}$, the bottom of the projected band gap is located just below the Fermi level, and both the existence of the $n=0$ surface state, not present within a jellium model of the surface, and the restricted available phase space play a key role in the determination of the $n=1$ image-state decay rate.

In Tables II and III we present the results of our calculations for the decay rate of the $n=1$ image state on $\mathrm{Cu}(100)$ and $\mathrm{Cu}(111)$, respectively, as obtained from Eq. (4) with use of MP wave functions in both Eqs. (6) and (11). First of all, we set all effective masses equal to the free-electron mass, and focus on the role that an accurate description of the

TABLE III. As in Table II, but for $\mathrm{Cu}(111)$ and together with the decay rate experimentally determined for this surface of copper (Ref. 17) at low temperature, $T=25 \mathrm{~K}$. Contributions to the linewidth from decay into bulk states lying below the bottom of the projected band gap, thereby excluding the contribution from decay into the $n=0$ intrinsic surface state, are displayed in parentheses.

\begin{tabular}{lclllll}
\hline \hline$m_{f}$ & $W$ & Bulk & Vacuum & Inter. & Total & Exp. \\
\hline$=1$ & SRM & $46(34)$ & $12(1)$ & $-22(-5)$ & $36(30)$ & \\
$=1$ & PZ & & $57(2)$ & & $57(2)$ & \\
$=1$ & $G W$-RPA & $44(28)$ & $47(5)$ & $-54(-12)$ & $37(21)$ & \\
$=1$ & $G W$-TDLDA & 43 & 42 & -45 & 40 & \\
$=1$ & $G W \Gamma$-TDLDA & 43.5 & 47 & -54 & 36.5 & \\
$\neq 1$ & $G W$-RPA & $32(24)$ & $34(5)$ & $-37(-12)$ & $29(17)$ & \\
$\neq 1$ & $G W \Gamma$-TDLDA & 30.5 & 35 & -38 & 28.5 & 30 \\
\hline \hline
\end{tabular}


screened interaction plays in the coupling of image states with the solid. Hence, we use three different models for the evaluation of $W\left(z, z^{\prime} ; \mathbf{q}_{\|}, \omega\right)$. First, the specular-reflection model (SRM) of Ritchie and Marusak ${ }^{37}$ has been considered, thereby assuming that bulk electrons are specularly reflected at the surface with no interference between ingoing and outgoing waves. Secondly, for the vacuum contribution to the decay rate $\left(z>0, z^{\prime}>0\right)$ the surface response function suggested by Persson and Zaremba ${ }^{38}$ (PZ) has been used. Finally, the screened interaction has been evaluated from Eq. (8) by solving the RPA integral equation [Eq. (10)] for the density-response function ( $G W$-RPA).

We note that simplified jellium models (SRM and PZ) for the evaluation of the screened interaction yield unrealistic results for the image-state lifetime. Bulk contributions to the linewidth are approximately well described within the specular reflection model, small differences resulting from an approximate description, within this model, of the so-called bregenzung effects. However, as within this model quantummechanical details of the surface are ignored, it fails to describe both vacuum and interference contributions to the decay rate. These quantum-mechanical details of the surface are approximately taken into account within the PZ jellium model, thereby resulting in a better approximation for the vacuum contribution to the decay rate than the SRM, but within the PZ model one neglects the coupling of the image state with the crystal that occurs through the penetration of the image-state wave function into the solid. Discrepancies between vacuum contributions obtained within this model and our more realistic full RPA calculations appear as a result of the jellium model of Ref. 38 being accurate only for $q_{\|} / q_{F}$ and $\omega / E_{F} \ll 1 \quad\left(q_{F}\right.$ is the Fermi momentum, i.e., $E_{F}$ $\left.=q_{F}^{2} / 2\right)$.

Now we look at the impact of short-range XC effects, which are well-known to reduce electron-electron interactions both between the image electron and the electron gas and between screening electrons themselves. Hence, we still set all effective masses equal to the free-electron mass, we introduce MP wave functions into Eqs. (6) and (11), and compare the $G W$-RPA calculations described above with the results that we obtain from either Eq. (8) $(G W)$ or Eq. (14) $(G W \Gamma)$ by using the density-response function of Eq. (15) with the LDA XC kernel of Eq. (17) (TDLDA). An inspection of the results presented in Tables I and II indicates that the existence of XC effects between screening electrons enhances the decay probability of image states. Nevertheless, this enhancement is more than compensated for by the large reduction of the decay rate produced by the presence of a XC hole around the image-state electron. Consequently, $G W$-RPA calculations produce decay rates that are larger than their more realistic $G W \Gamma$-TDLDA counterparts by no more than $\sim 5 \%$ in both (100) and (111) surfaces of $\mathrm{Cu}$.

Finally, we account for potential variation parallel to the surface through the introduction of a realistic effective mass. The dispersion $E_{0}\left(\mathbf{k}_{\|}\right)$of image states has been determined experimentally with the use of inverse photoemission techniques at off-normal emission, ${ }^{10}$ showing that the effective mass of image states in both $\mathrm{Cu}(100)$ and $\mathrm{Cu}(111)$ are $\sim 1$, i.e., the free-electron mass. Measurements of the dispersion of the $n=0$ surface resonance/state on the (100) and (111) surfaces of $\mathrm{Cu}$ have yielded effective masses of 0.50 and
0.42 , respectively, ${ }^{40-42}$ and for bulk states entering Eq. (6) we have chosen to increase the effective mass from our computed value of $m_{f}=0.40$ and 0.22 at the bottom of the gap in $\mathrm{Cu}(100)$ and $\mathrm{Cu}(111)$, respectively, to $m_{f}=1$ at the bottom of the valence band. As the effective mass of all available final states is smaller than the free-electron mass, the $n=1$ image-state decay rate of both $\mathrm{Cu}(100)$ and $\mathrm{Cu}(111)$ is found to be about $20-25 \%$ smaller than in the case of freeelectron behavior along the surface $\left(m_{f}=1\right)$. On the one hand, there is the effect of the decrease of the available phase space, which is easily found to scale as $\sqrt{m_{f}}$. On the other hand, as the effective mass decreases, the decay from the image state occurs, for a given energy transfer, through smaller parallel momentum transfer, which may result in either enlarged or diminished screened interactions, depending on the magnitude of momentum and energy transfers. Our results also indicate that $G W$-RPA and $G W \Gamma$-TDLDA calculations nearly coincide, as in the case of free-electron behavior along the surface, which is a consequence of the competition between $\mathrm{XC}$ effects associated to the image-state electron and to screening electrons themselves. $G W \Gamma$-TDLDA calculations, as obtained with use of realistic effective masses for the description of final-state wave functions, yield decay rates of the $n=1$ image state on $\mathrm{Cu}(100)$ and $\mathrm{Cu}(111)$ of 17 and $28.5 \mathrm{meV}$, respectively, in excellent agreement with the experimentally determined lifetimes ${ }^{43}$ of $40 \pm 6,{ }^{18,19}$ and $22 \pm 3$ fs. $^{17}$

With the aim of investigating the role that the various available bulk and surface states play in the decay of imagepotential states, now we focus on our full $G W$-RPA calculation of the $n=1$ image-state decay rate. Figure 5 exhibits $\tau_{f}^{-1}$ separate contributions to $\tau^{-1}$ from all final states lying below the projected band gap in $\mathrm{Cu}(100)$ (curves with circles) and $\mathrm{Cu}(111)$ (curves with squares), as obtained with the final-state effective mass set equal to the free-electron mass (solid lines) and with use of realistic values for $m_{f}$ (dashed lines). In the case of $\mathrm{Cu}(111)$, there is still a large contribution to $\tau^{-1}$ from the decay of the $n=1$ image state into the crystal-induced $n=0$ surface state, lying within the projected band gap, which approximately represents $40 \%$ of the total decay rate. Figures 5(a), 5(b), 5(c) and (d) exhibit bulk, vacuum, interference, and total contributions to $\tau_{f}^{-1}$. As the $n=1$ image-state wave-function overlap with the bulk is larger in $\mathrm{Cu}(111)$ than in the case of $\mathrm{Cu}(100)$, bulk contributions to $\tau_{f}^{-1}$ decay rates are much larger for $\mathrm{Cu}(111)$ than for $\mathrm{Cu}(100)$, as illustrated in Fig. 5(a). However, the large bulk-state overlap in $\mathrm{Cu}(111)$ is partially counterbalanced by the band gap extending on the (111) surface of $\mathrm{Cu}$ below the Fermi level. On the other hand, vacuum and interference contributions to the decay rate in both surfaces of $\mathrm{Cu}$ are comparable in magnitude and opposite in sign, yielding total decay rates that differ little from the bulk contribution. We also note that the total decay rate in $\mathrm{Cu}(100)$ (22 and $17.5 \mathrm{meV}$, with $m_{f}=1$ and $m_{f} \neq 1$, respectively) nearly coincides with the $\Sigma_{f} \tau_{f}^{-1}$ contribution from all bulk states in $\mathrm{Cu}(111)$ (21 and $17 \mathrm{meV}$ ). Hence, differences between total decay rates in $\mathrm{Cu}(100)$ and $\mathrm{Cu}(111)$ appear as a consequence of the presence of the $n=0$ surface state on $\mathrm{Cu}(111)$, which provides a key channel for the decay mechanism. 

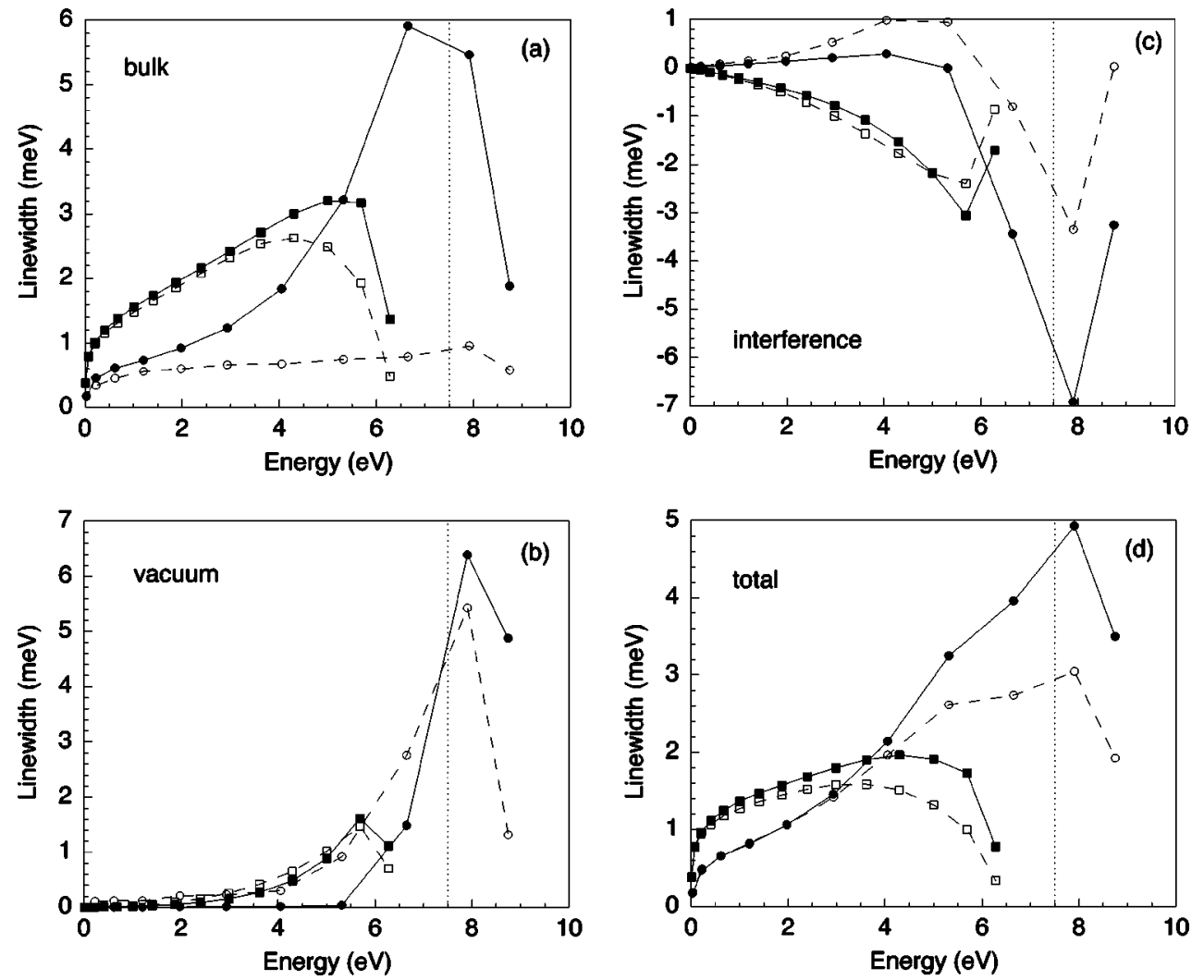

FIG. 5. (a) Bulk, (b) vacuum, (c) interference, and (d) total contributions to the $G W$-RPA damping rate $\tau^{-1}$ coming from the decay into the various $f$ available states lying below the projected band gap in $\mathrm{Cu}(100)$ (curves with circles) and $\mathrm{Cu}(111)$ (curves with squares), as obtained with the final-state effective mass set equal to the free-electron mass (solid lines) and with use of realistic values of $m_{f}$ (dashed lines). Model potential (MP) wave functions have been used for the description of all single-particle states entering Eqs. (4), (6), and (11). Vertical dotted lines represent the Fermi level.

Decay rates of image-potential states on $\mathrm{Cu}(100)$ with quantum number $n \leqslant 3$ are presented in Table IV, together with the experimentally determined lifetimes reported in Refs. 18 and 19. As before, the wave vector of the image state parallel to the surface, $\mathbf{k}_{11}$, has been set equal to zero, all the wave functions entering Eqs. (4), (6), and (11) have

TABLE IV. Calculated decay rates, in linewidth units (meV), and lifetimes, in femtoseconds ( $\mathrm{fs}$ ), of the $n \leqslant 3$ image states on $\mathrm{Cu}(100)$, together with the experimentally determined lifetimes of Refs. 18 and 19. In the case of $n=2$ and $n=3$, contributions from decay into the lower lying image states are also displayed. The screened interaction has been evaluated within the $G W$-RPA. Model potential (MP) wave functions have been used for the description of all single-particle states entering Eqs. (4), (6), and (11). The effective mass has been set equal to either the free-electron mass or to the realistic values described in the text. The result of introducing into Eq. (6) a realistic effective mass for all available final states is displayed in parentheses.

\begin{tabular}{lccc}
\hline \hline & Linewidths & Lifetimes & Experiment \\
\hline$n=1$ & $22(17.5)$ & $30(38)$ & $40 \pm 6$ \\
$n=2$ & $5(3.9)$ & $132(168)$ & $120 \pm 15$ \\
$n=2$ to $n=1$ & $0.5(0.4)$ & & \\
$n=3$ & $1.8(1.4)$ & $367(480)$ & $300 \pm 20$ \\
$n=3$ to $n=2$ & $0.05(0.05)$ & & \\
$n=3$ to $n=1$ & $0.17(0.16)$ & & \\
\hline \hline
\end{tabular}

been chosen to be MP wave functions, and the screened interaction has been evaluated within the $G W$-RPA. Decay rates of the $n=2$ and $n=3$ image states have been split as follows:

$$
\tau^{-1}=\sum_{f} \tau_{f}^{-1}+\tau_{n=1}^{-1}
$$

and

$$
\tau^{-1}=\sum_{f} \tau_{f}^{-1}+\tau_{n=1}^{-1}+\tau_{n=2}^{-1},
$$

respectively, where $\Sigma_{f} \tau_{f}^{-1}$ represents, as in the case of the $n=1$ image state, the decay rate from transitions to all available states lying below the projected band gap, and $\tau_{n}^{-1}$ represents the contribution from decay into the lower lying $n$ image state. We observe that lower lying image states can give noticeable contributions to the decay rate of excited, i.e., $n=2$ and $n=3$ image states. Decay of these states into the $n=1$ image state results in linewidths that represent $\sim 10 \%$ of the total linewidth. Decay of the $n=3$ image state into the $n=2$ lower lying state results in a linewidth that represents $\sim 3 \%$ of the total linewidth. We also observe that both our calculated and the experimentally determined lifetimes of image states in $\mathrm{Cu}(100)$ increase with $n$ as $\sim 1 / n^{3}$, in agreement with previous theoretical predictions. ${ }^{1}$ Discrepancies between our calculated inelastic lifetimes of excited image states $(n>1)$ and experimental measurements may be 
attributed to scattering with phonons, which occurs on a time scale ( $\sim 1 \mathrm{ps})$ that is comparable for these states to the electron-electron relaxation time.

\section{SUMMARY}

We have reported extensive self-consistent calculations of the imaginary part of the electron self-energy and the decay rate of image states on the (100) and (111) surfaces of $\mathrm{Cu}$, by going beyond a free-electron description of the metal surface. We have found that the imaginary part of the electron self-energy outside the surface is highly nonlocal, and have found the magnitude of the maximum of this quantity to reach its highest value near the surface. We have presented the results of calculations of the lifetime of the first three image states on $\mathrm{Cu}(100)$ and the first image state on $\mathrm{Cu}(111)$, and have focused on the impact of band-structure and manybody effects on these quantities. We have found that bandstructure effects on the evaluation of final-state wave functions may be approximately accounted for through introduction of the restriction that only final states lying below the projected band gap are allowed, and the impact of the band structure through the evaluation of the screened interaction has been found not to be large. We have shown that simplified jellium models for the electronic response yield unrealistic results for the lifetime of image states, thereby a detailed description of the screened interaction being of crucial importance in the understanding of the origin of image-state lifetimes. We have evaluated the screened interaction within three different self-consistent many-body schemes, depending on whether XC effects are not included ( $G W$-RPA) or they are included through the introduction of a XC hole around screening electrons only (GW-TDLDA) or around both the image-state electron and screening electrons ( $G W \Gamma$-TDLDA). We have reached the important conclusion that $G W$-RPA calculations produce decay rates that are very close to $G W \Gamma$-TDLDA calculations, which are obtained with full inclusion of XC effects. With the use of either the free-electron mass or more realistic effective masses for all final states, decay rates of the $n=1$ image state on $\mathrm{Cu}(100)$ are found to be smaller than those of the $n=1$ image state on $\mathrm{Cu}(111)$. The large bulk-state overlap on $\mathrm{Cu}(111)$ is found to be approximately counterbalanced by the band gap extending on the $\mathrm{Cu}(111)$ surface below the Fermi level, and differences between decay rates on the (100) and (111) surfaces of $\mathrm{Cu}$ are found to be mainly due to the large contribution, in the case of $\mathrm{Cu}(111)$, from decay into the crystal-induced $n=0$ surface state located within the projected band gap. The results we have obtained for the $n$ $=1$ image-state lifetime on both surfaces of $\mathrm{Cu}$ with use of realistic effective masses, which are $\sim 20 \%$ below those obtained with $m_{f}=1$, are in excellent agreement with experimentally determined decay times.

\section{ACKNOWLEDGMENTS}

The authors gratefully acknowledge partial support by the University of the Basque Country, the Basque Hezkuntza, Unibertsitate eta Ikerketa Saila, the Spanish Ministerio de Educación y Cultura, and Iberdrola S. A.
${ }^{1}$ P. M. Echenique and J. B. Pendry, J. Phys. C 11, 2065 (1978); P. M. Echenique and J. B. Pendry, Prog. Surf. Sci. 32, 111 (1990).

${ }^{2}$ R. M. Osgood, Jr. and X. Wang, Solid State Phys. 51, 1 (1997).

${ }^{3}$ V. Dose, W. Altmann, A. Goldmann, U. Kolac, and J. Rogozik, Phys. Rev. Lett. 52, 1919 (1984).

${ }^{4}$ D. Straub and F. J. Himpsel, Phys. Rev. Lett. 52, 1922 (1984); D. Straub and F. J. Himpsel, Phys. Rev. B 33, 2256 (1986).

${ }^{5}$ J. B. Pendry, Phys. Rev. Lett. 45, 1356 (1980).

${ }^{6}$ P. D. Johnson and N. V. Smith, Phys. Rev. B 27, 2527 (1983).

${ }^{7}$ K. Giesen, F. Hage, F. J. Himpsel, H. J. Riess, and W. Steinmann, Phys. Rev. Lett. 55, 300 (1985).

${ }^{8}$ D. F. Padowitz, W. R. Merry, R. E. Jordan, and C. B. Harris, Phys. Rev. Lett. 69, 3583 (1992).

${ }^{9}$ S. Schuppler, N. Fischer, Th. Fauster, and W. Steinmann, Phys. Rev. B 46, 13539 (1992).

${ }^{10} \mathrm{Th}$. Fauster and W. Steinmann, in Electromagnetic Waves: Recent Developments in Research, Photonic Probes of Surfaces, Vol. 2, edited by P. Halevi (Elsevier, Amsterdam, 1995).

${ }^{11}$ C. B. Harris, N.-H. Ge, R. L. Lingle, Jr., J. D. McNeill, and C. M. Wong, Annu. Rev. Phys. Chem. 48, 711 (1997).

${ }^{12}$ R. W. Schoenlein, J. G. Fujimoto, G. L. Eesley, and T. W. Capehart, Phys. Rev. Lett. 61, 2596 (1988).

${ }^{13}$ R. W. Schoenlein, J. G. Fujimoto, G. L. Eesley, and T. W. Capehart, Phys. Rev. B 41, 5436 (1990).

${ }^{14}$ R. W. Schoenlein, J. G. Fujimoto, G. L. Eesley, and T. W. Capehart, Phys. Rev. B 43, 3688 (1991).

${ }^{15}$ T. Hertel, E. Knoesel, M. Wolf, and G. Ertl, Phys. Rev. Lett. 76, 535 (1996)
${ }^{16}$ M. Wolf, E. Knoesel, and T. Hertel, Phys. Rev. B 54, 5295 (1996); M. Wolf, Surf. Sci. 377-379, 343 (1997).

${ }^{17}$ E. Knoesel, A. Hotzel, and M. Wolf, J. Electron Spectrosc. Relat. Phenom. 88-91, 577 (1998).

${ }^{18}$ U. Höffer, I. L. Shumay, Ch. ReuB, U. Thomann, W. Wallauer, and Th. Fauster, Science 227, 1480 (1997).

${ }^{19}$ I. L. Shumay, U. Höfer, Ch. ReuB, U. Thomann, W. Wallauer, and Th. Fauster, Phys. Rev. B 58, 13974 (1998)

${ }^{20}$ P. M. Echenique, F. Flores, and F. Sols, Phys. Rev. Lett. 55, 2348 (1985).

${ }^{21}$ P. L. de Andrés, P. M. Echenique, and F. Flores, Phys. Rev. B 39, 10356 (1989).

${ }^{22}$ S. Gao and B. I. Lundqvist, Suppl. Prog. Theor. Phys. 106, 405 (1991).

${ }^{23}$ J. Osma, I. J. Sarria, E. V. Chulkov, J. M. Pitarke, and P. M. Echenique, Phys. Rev. B 59, 10591 (1999).

${ }^{24}$ E. V. Chulkov, I. Sarria, V. M. Silkin, J. M. Pitarke, and P. M. Echenique, Phys. Rev. Lett. 80, 4947 (1998).

${ }^{25}$ E. V. Chulkov, J. Osma, I. Sarria, V. M. Silkin, and J. M. Pitarke, Surf. Sci. 433, 882 (1999).

${ }^{26}$ L. Hedin and S. Lundqvist, Solid State Phys. 23, 1 (1969).

${ }^{27}$ See, e.g., D. Pines, Elementary Excitations in Solids (Addison Wesley, New York, 1963), Chap. 3.

${ }^{28}$ G. D. Mahan and B. E. Sernelius, Phys. Rev. Lett. 62, 2718 (1989).

${ }^{29}$ G. D. Mahan, Many-Particle Physics, 2nd ed. (Plenum, New York, 1990). 
${ }^{30}$ A. G. Eguiluz, Phys. Rev. Lett. 51, 1907 (1983); Phys. Rev. B 31, 3303 (1985).

${ }^{31}$ P. Hohenberg and W. Kohn, Phys. Rev. 136, B864 (1964); W. Kohn and L. J. Sham, Phys. Rev. 140, A11 333 (1965).

${ }^{32}$ R. M. Dreizler and E. K. U. Gross, Density Functional Theory, an Approach to the Quantum Many Body Problem (Springer, Berlin, 1990).

${ }^{33}$ We use the parametrization of Perdew and Zunger [J. P. Perdew and A. Zunger, Phys. Rev. B 23, 5048 (1981)] for $E_{x c}[n]$, which is based on Monte Carlo calculations by Ceperley and Alder [D. M. Ceperley and B. J. Alder, Phys. Rev. Lett. 45, 1196 (1980)].

${ }^{34}$ A. Zangwill and P. Soven, Phys. Rev. A 21, 1561 (1980).

${ }^{35}$ E. V. Chulkov. V. M. Silkin, and P. M. Echenique, Surf. Sci. 391, L1217 (1997).

${ }^{36}$ S. L. Hulbert, P. D. Johnson, M. Weinert, and R. F. Garrett, Phys. Rev. B 33, 760 (1986).
${ }^{37}$ R. H. Ritchie and A. L. Marusak, Surf. Sci. 4, 234 (1966); P. M. Echenique, F. J. García de Abajo, V. H. Ponce, and M. E. Uranga, Nucl. Instrum. Methods Phys. Res. B 96, 583 (1995).

${ }^{38}$ B. N. J. Persson and E. Zaremba, Phys. Rev. B 31, 1863 (1985). For copper surfaces, the $m_{o p t}, h$, and $\xi$ parameters are taken to be $1.68,1.84$, and 0.49 , respectively.

${ }^{39}$ J. J. Deisz. A. G. Eguiluz, and W. Hanke, Phys. Rev. Lett. 71, 2793 (1993).

${ }^{40}$ S. D. Kevan, Phys. Rev. Lett. 50, 526 (1983).

${ }^{41}$ A. Goldmann, V. Dose, and G. Borstel, Phys. Rev. B 32, 1971 (1985).

${ }^{42}$ S. L. Hulbert, P. D. Johnson, N. G. Stoffel, W. A. Royer, and N. V. Smith, Phys. Rev. B 31, 6815 (1985).

${ }^{43}$ The decay rate of Eq. (4) is related to the lifetime, $\tau$, by $\tau^{-1} \tau$ $=1$ a.u. $=658 \mathrm{meV}$ fs 